Title:

PHYSICAL NATURE OF THE LOW-SPEED SOLAR WIND

Author(s):

J. T. Gosling

RECENED

Ju1 19 ต9gs

OSTI

Submitted to:

Scientific Basis for Robotic Explorations Close to the Sun

DISTRIBUTION OF THIS DOCUMENT IS UNLMUTED Ph

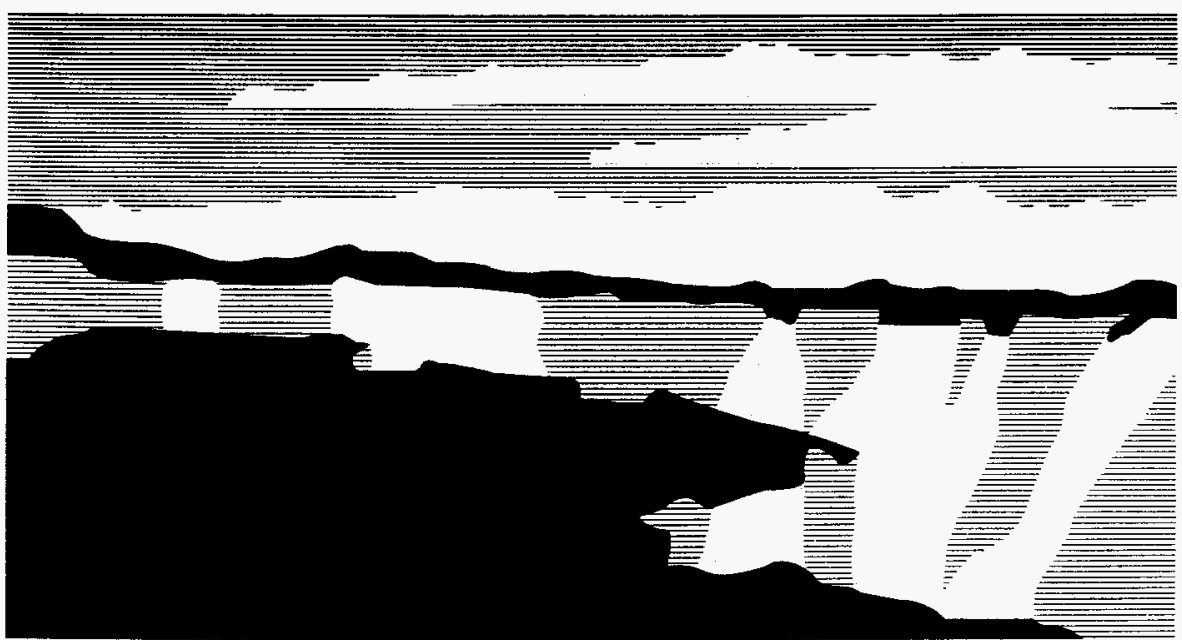

Los Alamos National Laboratory, an affirmative action/equal opportunity empioyer, is operated by the University of California for the U.S. Department of Energy under contract W-7405-ENG-36. By acceptance of this article, the publisher recognizes that the U.S. Government retains a nonexclusive, royalty-free license to publish or reproduce the published form of this contribution, or to allow others to do so, for U.S. Government purposes. The Los Alamos National Laboratory requests that the publisher identify this articie as work performed under the auspices of the U.S. Department of Energy. 


\section{DISCLAIMER}

Portions of this document may be illegible in electronic image products. Images are produced from the best available original document. 


\section{DISCLAIMER}

This report was prepared as an account of work sponsored by an agency of the United States Government. Neither the United States Government nor any agency thereof, nor any of their employees, makes any warranty, express or implied, or assumes any legal liability or responsibility for the accuracy, completeness, or usefulness of any information, apparatus, product, or process disclosed, or represents that its use would not infringe privately owned rights. Reference herein to any specific commercial product, process, or service by trade name, trademark, manufacturer, or otherwise does not necessarily constitute or imply its endorsement, recommendation, or favoring by the United States Government or any agency thereof. The views and opinions of authors expressed herein do not necessarily state or reflect those of the United States Government or any agency thereof. 


\title{
Physical Nature of the Low-Speed Solar Wind
}

\author{
J. T. Gosling \\ Los Alamos National Laboratory \\ Los Alamos, New Mexico 87545
}

\begin{abstract}
In situ observations indicate that the low-speed wind is highly variable. It commonly originates on open field lines that thread coronal streamers in the vicinity of the magnetic equator, but transient ejections are also a source of low-speed flows on occasion. Close to the Sun a large flow shear probably is common at the interface between low- and high-speed flows. Near solar activity minimum low-speed flows are confined to a narrow band $40-45^{\circ}$ wide centered roughly on the solar equator, but near solar maximum low-speed flows may dominate at all heliographic latitudes.
\end{abstract}

In the inner heliosphere at low heliographic latitudes the solar wind tends to be organized into alternating streams of high- and low-speed flows [1]. It is well established that high-speed flows in the quiescent (i.e., non-transient) wind usually originate in coronal holes that extend equatorward from the magnetic poles of the Sun [2]. On the other hand, the origin of the low-speed wind is less certain and it is not entirely clear that the low-speed wind ever is truly quiescent. The base, or minimum, solar wind expansion speed at $1 \mathrm{AU}$ is $\sim 280 \mathrm{~km} \mathrm{~s}^{-1}$, and the solar wind observed near Earth tends to return towards this base value, although seidom actually reaching it, after each excursion to higher speed. The low-speed wind usually has a distinctly different physical character from that of the high-speed wind, presumably reflecting the fact that it originates from a different region in the solar atmosphere. Our purpose here is to provide a brief overview of the physical nature of the low-speed wind, primarily as inferred from in situ observations obtained near the ecliptic plane.

Figure 1 displays selected solar wind plasma and magnetic field parameters from IMP 7 and 8 obtained during a 37-day interval in 1974 on the declining phase of the solar activity cycle. During this interval two high-speed streams were observed each solar rotation; these originated alternately from equatorial extensions of the coronal holes in the northern and southern polar regions. Between the high-speed streams the wind speed returned to values approaching $300 \mathrm{~km} \mathrm{~s}^{-1}$. As compared to the high-speed wind, the low-speed wind was characterized by a generally high and variable density, by a low and variable helium abundance (relative to $\mathrm{H}^{+}$), and by polarity reversals in the interplanetary magnetic field, IMF. The latter can be distinguished by $\sim 180^{\circ}$ changes in $\phi_{\mathrm{B}}$, the azimuthal angle of the IMF.

Table 1, which contrasts values for selected solar wind parameters in the high- 


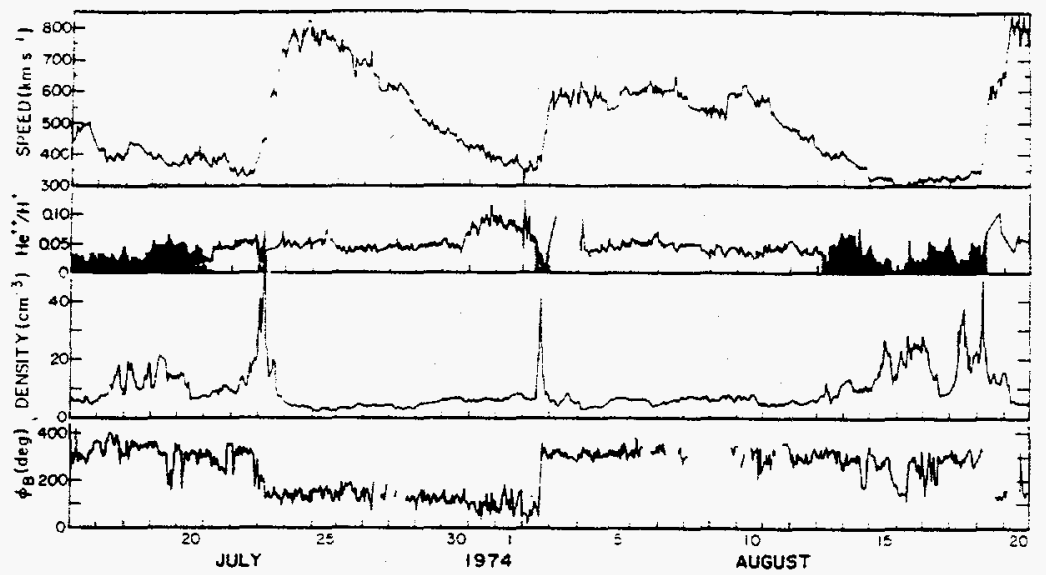

Figure 1. Wind flow speed, helium abundance, proton density and magnetic field azimuth in July and August 1974. From [3].

and low-speed wind based on measurements from the IMP spacecraft over the years 1971-76, confirms that the differences between the high- and low-speed wind evident in Figure 1 are generic. Not only are long-term average values of density, flow speed, number flux, proton and electron temperatures, and alpha/proton temperature ratio, quite different in the low- and high-speed wind, but the \% variances in all of these quantities except speed and in the helium abundance are considerably higher in the low-speed wind than in the high-speed wind [5]. This constitutes strong evidence that the slow wind originates in distinctly different regions in the solar atmosphere and perhaps distinctly different processes from that of the fast wind. It is not presently known if the high variability of the low-speed wind is primarily a consequence of spatial structure in the source region and solar rotation, or whether it is primarily associated with temporal variability in the outflow, or some combination thereof. Moreover, it is uncertain where the open field lines populating the slow wind originate on the solar surface.

When observations like those in Figure 1 are mapped back to the Sun it is found that the low-speed wind appears to originate in or near the coronal streamer belt that wraps around the Sun at low heliographic latitudes [3]. Sharp field polarity

TABLE 1. Plasma characteristics of high-and low-speed wind.*

\begin{tabular}{clllllll} 
& \multicolumn{3}{c}{ High Speed } & \multicolumn{3}{c}{ Low Speed } \\
Parameter & Mean & $\sigma$ & \%Var. & Mean & $\sigma$ & \%Var. \\
\hline $\mathrm{N}\left(\mathrm{cm}^{-3}\right)$ & 3.9 & 0.6 & 15 & 11.9 & 4.5 & 38 \\
$\mathrm{~V}\left(\mathrm{~km} \mathrm{~s}^{-1}\right)$ & 702 & 32 & 5 & 327 & 15 & 5 \\
$\mathrm{NV}\left(\mathrm{cm}^{-2} \mathrm{~s}^{-1}\right)$ & $2.7 \mathrm{e} 8$ & $0.4 \mathrm{e} 8$ & 15 & $3.9 \mathrm{e} 8$ & $1.5 \mathrm{e} 8$ & 38 \\
$\mathrm{~T}_{\mathrm{p}}(\mathrm{K})$ & $2.3 \mathrm{e} 5$ & $0.3 \mathrm{e} 5$ & 13 & $.34 \mathrm{e} 5$ & $.15 \mathrm{e} 5$ & 44 \\
$\mathrm{~T}_{\mathrm{e}}(\mathrm{K})$ & $1.0 \mathrm{e} 5$ & $.1 \mathrm{e} 5$ & 8 & $1.3 \mathrm{e} 5$ & $.3 \mathrm{e} 5$ & 20 \\
$\mathrm{~T}_{\alpha} / \mathrm{T}_{\mathrm{p}}$ & 6.2 & 1.3 & 21 & 3.2 & 0.9 & 28 \\
$\mathrm{~N}_{\alpha}$ & .048 & .005 & 10 & .047 & .019 & 40 \\
$*$ & & & & & &
\end{tabular}


reversals, such as that on August 2, 1974 in Figure 1, appear to correspond to regions on the Sun where the streamer belt and the magnetic equator, which threads roughly through its center, are strongly inclined in the north-south direction. In contrast, multiple density peaks and multiple polarity reversals, such as in the August 13-19 interval, tend to be associated with regions where the streamer belt is aligned roughly parallel to the solar equator and is centered approximately at the same latitude as Earth. These associations suggest that the multiplicity of density peaks and field polarity reversals often observed in the low-speed wind near Earth are, at least at times, a consequence of spatial structure in and warping of the streamer belt and its embedded current sheet. However, some of the structure may be associated with temporal variability back at the Sun. In addition, it has been suggested [6] that multiple field polarity reversals may be evidence that multiple current sheets often extend out into the solar wind from coronal streamers.

The structure of the solar wind evolves with increasing heliocentric distance as high-speed streams steepen and overtake and compress the low-speed wind that lies ahead[7,8]. Effects of this evolution can be readily discerned in Figure 1 in the steepened stream speed profiles and in the compressions that occur on the rising speed portions of the streams. Such effects need to be considered when comparing models with actual observations of the slow wind at any distance from the Sun. (Compressions and rising speed gradients were specifically excluded in compiling Table 1.) Fast-slow wind interaction effects, although significant even at $1 \mathrm{AU}$, are even more pronounced farther out in the solar system. For example, the minimum solar wind speed at Jupiter's orbit typically is of the order of $400 \mathrm{~km} \mathrm{~s}^{-1}$ because of such interactions.

We have already provided evidence that low- and high-speed flows have distinctly different origins in the solar atmosphere. Further evidence for distinctly different origins can be found at solar wind boundaries known as stream interfaces. A stream interface $[9,10]$ is identified in solar wind plasma data by a large drop in plasma density simultaneous with a large increase in proton temperature that occurs on the rising portion of a high-speed stream, i.e., within the compression where fast wind overtakes slow wind. For a subset of all stream interfaces these changes occur discontinuously in the data. Figure 2 shows superposed epoch plots at two temporal resolutions for a set of 23 discontinuous stream interfaces identified in the IMP plasma data. It is apparent from the left portion of the figure that (1) the proton pressure (resulting from compression) is a maximum at the interface; (2) the speed jumps discontinuously at the interface and the speed gradient changes discontinuously there; and (3) the azimuthal flow angle changes abruptly at the interface. Together, these and other observations not shown here indicate that the interface is a boundary separating what was originally (close to the Sun) slow, cold, dense plasma from what was originally fast, hot, rare plasma [11]. From the right portion of the figure it is also apparent that, on average, a jump in helium abundance occurs at the interface. This is further evidence that the slow and fast wind arise from distinctly different regions in the solar atmosphere.

Measurements made by the SWICS instrument on Ulysses [12] also reveal 

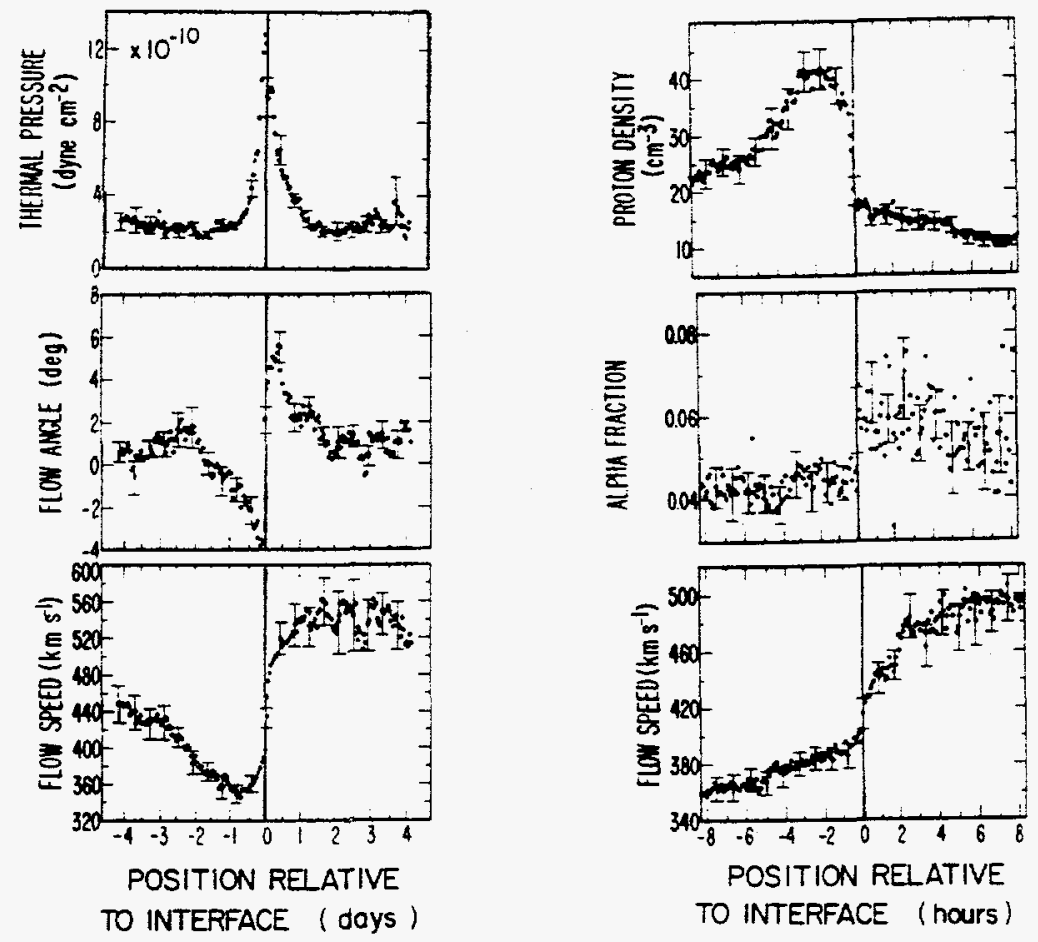

Figure 2. Superposed epoch plots of selected solar wind parameters using 1 -hr averages (left) and 5-min averages (right) for 23 abrupt stream interfaces. Adapted from [11].

distinct differences in abundances and ionic temperatures in low- and high-speed flows. When compared to the high-speed wind, the low speed wind is distinguished by higher $\mathrm{O}$ and $\mathrm{C}$ freezing-in temperatures and by higher abundance ratios of $\mathrm{Mg} / \mathrm{O}$ and $\mathrm{Fe} / \mathrm{O}$. The cadence of the SWICS instrument measurements is quite low, and it is presently uncertain whether or not the above changes on the rising speed portions of the streams occur precisely at the stream interfaces. Nevertheless, the data clearly establish that the high- and low-speed flows must have distinctly different coronal origins.

It has long been known that when solar wind measurements are mapped back to the Sun by assuming constant speed in transit that the trailing portions of highspeed streams commonly map to nearly fixed solar longitudes $[11,13,14]$. This suggests that very large flow shears commonly exist at the boundaries between low and high speed flows very close to the Sun. (The mapping does not work on the rising speed portions of streams because of the significant interplanetary plasma accelerations and decelerations that occur there. Nevertheless, the common presence of discontinuous or nearly discontinuous stream interfaces on the leading edges of many high-speed streams also suggests the presence of very large flow shears at the boundaries between low- and high-speed flows close to the Sun.) It is not presently known exactly where this region of high flow shear is in the corona. One suggestion [11] is that the shear occurs at the outer edges of visible 

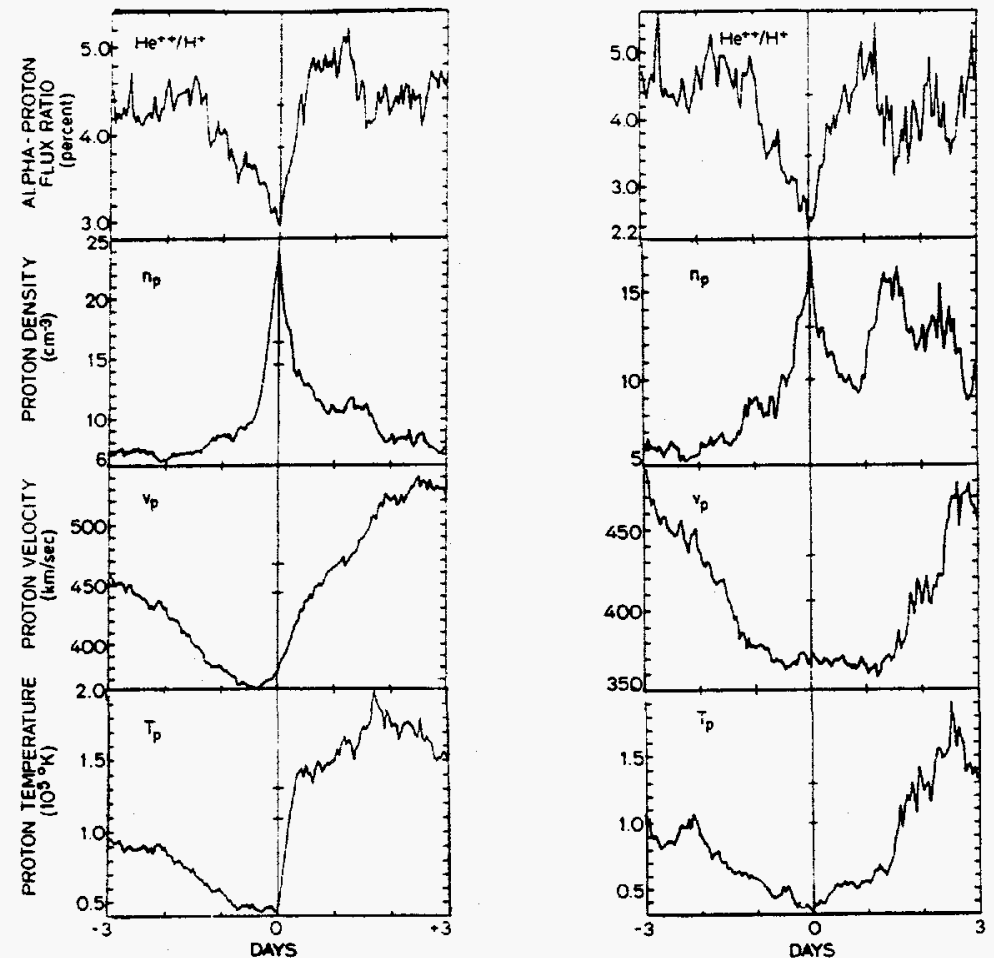

Figure 3. Superposed epoch plots of selected solar wind parameters for 74 "clean" crossings of the HCS (left) and for a subset of 23 crossings where the HCS had not been swept into a stream compression region (right). Adapted from [16].

coronal streamers. Another possibility is that the shear occurs at the boundaries of coronal holes. Indeed, coronal hole boundaries and the outer edges of coronal streamers may be one and the same.

The heliospheric current sheet, HCS, which represents the interplanetary extension of the solar magnetic equator and which separates interplanetary space into regions of opposite magnetic polarity, also organizes solar wind plasma observations [15] (for example, see Figure 1). The left portion of Figure 3 shows the result of a superposed epoch analysis of 74 relatively clean (i.e., sharp) HCS crossings observed by IMP [16]. A maximum in plasma density, and minimums in flow speed, proton temperature, and helium abundance occur, on average, at the HCS. The overall "signal" associated with the HCS is about 5 days wide on the average. If interpreted strictly as a solar longitudinal effect associated with solar rotation (this may not be entirely valid), the average HCS signal has a longitudinal extent of $\sim 65^{\circ}$.

The plasma signature associated with the HCS in the left portion of Figure 3 is affected by stream evolution effects in interplanetary space since the $\mathrm{HCS}$ at $1 \mathrm{AU}$ often lies within the compression region formed by stream steepening. In order to minimize such effects, a set of 23 sharp HCS crossings were identified in the IMP data where the HCS had not been overtaken by the stream compression [16]. The 
result of a superposed epoch analysis of that special set of crossings is shown in the right portion of Figure 3. Here the average plasma signature is more symmetrical about the HCS and is still $\sim 5$ days wide. In addition, two separate density peaks can be distinguished. One of these (the first) appears to be an intrinsic signature associated with the HCS, while the second peak appears to be associated with compression in interplanetary space since it coincides with a rising speed gradient. With regard to Figure 3 it should be emphasized that the coincidence of minimums in helium abundance, solar wind speed, and proton temperature and a maximum in proton density with the HCS is an average effect not always exhibited by individual events included in the analysis [3]. For example, the high density region associated with the HCS is often offset to one side of the HCS and can be considerably narrower than Figure 3 would suggest [17]. More recent analyses also indicate that fluctuations in plasma density and magnetic field strength are enhanced in the vicinity of the HCS [18], presumably in accord with interplanetary scintillation measurements in the vicinity of the HCS made considerably closer to the Sun.

To this point our emphasis has been on the low-speed wind as it is observed in the ecliptic plane on the declining phase of the solar activity cycle and near solar activity minimum. Evidence suggests that at such times the low-speed wind is observed only in the vicinity of the heliospheric current sheet. Since the HCS is confined to low heliographic latitudes during these phases of the solar cycle, so too is the low-speed wind. Figure 4, which provides an overview of solar wind speed and density observed by Ulysses during its recent (relatively) fast latitude scan from $-80^{\circ}$ heliographic latitude to $+80^{\circ}$ near solar minimum [19], helps emphasize this point. During this latitude scan virtually all of the major variations in the solar wind flow, including excursions to low speed, were confined to a band, centered slightly below the solar equator, whose total width in heliographic latitude was $\sim 43^{\circ}$ [20]. At higher latitudes a nearly uniform high-speed wind was observed.

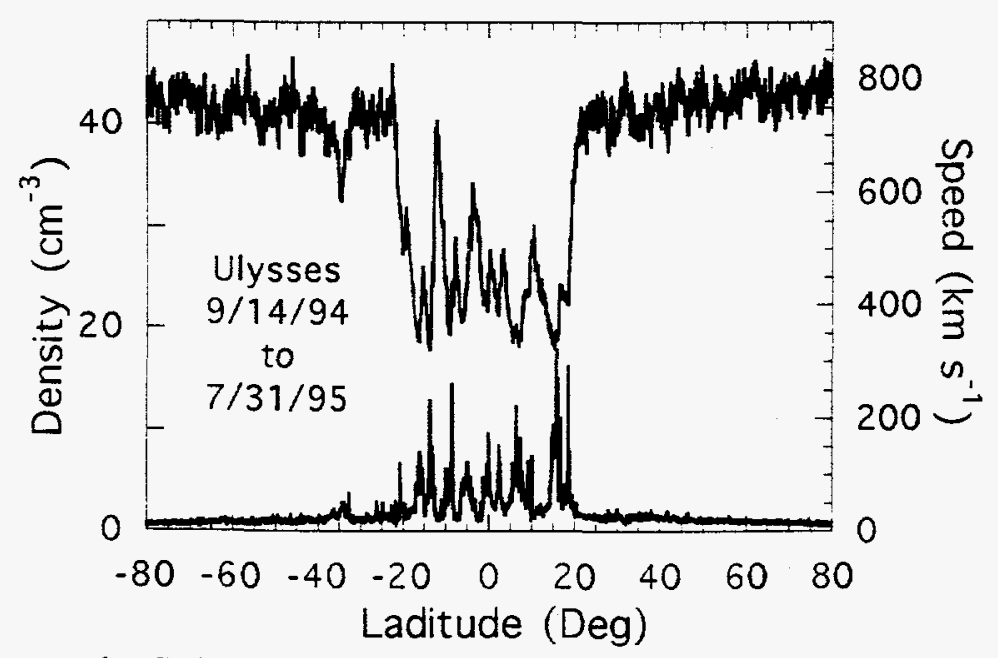

Figure 4. Solar wind speed and density measured by Ulysses during its rapid transit from $-80^{\circ}$ to $+80^{\circ}$ latitude during 1994,95 . 
This result is in substantial agreement with determinations of the 3-dimensional flow pattern near solar minimum obtained from interplanetary scintillation, IPS, measurements [21]. Moreover, the IPS measurements indicate that low-speed wind is much more pervasive at high heliographic latitudes near solar activity maximum when the polar coronal holes shrink in size and when much of the corona is constrained from expanding by the strong coronal magnetic fields present then. Thus near solar maximum a low-speed wind may be the rule at all heliographic latitudes rather than the exception and may not necessarily be confined to the vicinity of the heliospheric current sheet.

We have noted that temporal variations back at the Sun are probably one source of variability in the low-speed wind as observed near Earth. The most spectacular temporal variations that occur in the corona are coronal mass ejections, or CMEs $[22,23]$. As observed by coronagraphs within $\sim 5$ solar radii of Sun center CMEs have outward speeds ranging from less than $100 \mathrm{~km} \mathrm{~s}^{-1}$ in some of the slower events up to at least $2000 \mathrm{~km} \mathrm{~s}^{-1}$ in some of the faster events [24-26]. Approximately half of the CMEs observed by the coronagraph on the Solar Maximum Mission satellite had speeds less than $300 \mathrm{~km} \mathrm{~s}^{-1}$, which is close to the minimum solar wind speed in the ecliptic plane at $1 \mathrm{AU}$. Yet all of these events appeared to have escaped from the Sun. This indicates that slow CMEs observed in the corona receive substantial further accelerations beyond 5 solar radii as they travel out into interplanetary space. We have argued elsewhere [27] that slow CMEs are accelerated outward by essentially the same processes that accelerate the normal solar wind (i.e., by the overall outward pressure gradient) and that acceleration profiles of slow CMEs close to the Sun probably mimic that of the normal slow solar wind.

In closing, it is useful to note a number of questions provoked by in situ observations of the slow solar wind. These include the following:

1. Why are there two (fast and slow), and only two, fundamental solar wind flow states and why is the mass flux for these two flow states nearly the same?

2. Where does the slow wind originate in the corona and what is(are) the acceleration process(es)?

3. How does the acceleration process relate to coronal temperature and flux tube divergence?

4. Why is the minimum solar wind speed far from the Sun about $280 \mathrm{~km} \mathrm{~s}^{-1}$ ?

5. What is the origin of the large variability of the slow wind?

6. Why is the Helium abundance so variable in the slow wind and why does it minimize, on average, at the heliospheric current sheet?

7. What is the structure of the heliospheric current sheet close to the Sun?

8. Does reconnection occur commonly at the HCS and what is the relationship between such reconnection and heat flux dropouts and double ion beams?

9. What sort of velocity shears exist at the slow/fast wind interface close to the Sun and how does that interface relate to observed coronal structure?

10. Why and how do slow CMEs attain the same minimum speed as the normal solar wind? 


\section{REFERENCES}

1.Snyder, C. W., Neugebauer, M., and Rao, U.R., J. Geophys. Res. 68, 6361 (1963).

2. Krieger, A. S., Timothy, A. F., and Roelof, E. C., Solar Phys. 29, 505 (1973).

3. Gosling, J. T., Borrini, G., Asbridge, J. R., Bame, S. J., Feldman, W. C., and Hansen, R. T., J. Geophys. Res. 86, 5438 (1981).

4. Feldman, W. C., Asbridge, J. R., Bame, S. J., and Gosling, J. T., in The Solar Output and Its Variation, edited by O. R. White, Boulder: Colorado Assoc. Univ. Press, 1977, pp. 351-381.

5. Bame, S. J., Asbridge, J. R., Feldman. W. C., and Gosling, J. T., J. Geophys. Res. 82, 1487 (1977).

6. Crooker, N. U., Siscoe, G. L., Shodhan, S., Webb, D. F., and Gosling, J. T., J. Geophys. Res. 98, 9371 (1993).

7. Gosling, J. T., Hundhausen, A. J., Pizzo, V., and Asbridge, J. R., J. Geophys. Res. 77, 5442 (1972).

8. Hundhausen, A. J., Coronal Expansion and Solar Wind, New York: SpringerVerlag, 1972, 238 pgs.

9. Belcher, J. W., and Davis, L., J. Geophys. Res. 76, 3534, (1971).

10. Burlaga, L. F., J. Geophys. Res. 79, 3717 (1974).

11. Gosling, J. T., Asbridge, J. R., Bame, S. J., and Feldman, W. C., J. Geophys. Res. 83, 1401, (1978).

12. Geiss, J., Gloeckler, G., von Steiger, R., et al., Science, 268, 1033 (1995).

13. Roelof, E. C., and Krimigis, S. M., J. Geophys. Res. 78, 5375 (1973).

14. Nolte, J. T., Krieger, A. S., Timothy, A. F., et al., Solar Phys. 46, 303 (1976).

15. Wilcox, J. M., and Ness, N. F., J. Geophys. Res. 70, 5793 (1965).

16. Borrini, G., Gosling, J. T., Bame, S. J., Feldman, W. C., and Wilcox, J. M., J. Geophys. Res. 86, 4565 (1981).

17. Winterhalter, D. , Smith, E. J., Burton, M. E., Murphy, N. and McComas, D. J., $J$. Geophys. Res. 99, 6667 (1994).

18. Huddleston, D., Woo, R., and Neugebauer, M., J. Geophys. Res. 100, 19,951 (1995).

19. Phillips, J.L., Bame, S. J., Barnes, A., et al., Geophys. Res. Lett. 22, 3301 (1995).

20. Gosling, J. T., Bame, S. J., Feldman, W. C., et al., Geophys. Res. Lett. 22, 3329 (1995).

21. Coles, W. A., Space Sci. Rev. 72, 211 (1995).

22. Kahler, S., Revs. Geophys. 25, 663 (1987).

23. Gosling, J. T., J. Geophys. Res. 98, 18,937 (1993).

24. Gosling, J. T., Hildner, E. MacQueen, R. M., Munro, R. H., Poland, A. I., and Ross, C. L., Solar Phys. 48, 389 (1976).

25. Howard, R., Sheeley, N. R., Koomen, M. J., and Michels, D. J., J.

Geophys. Res. 90, 8173 (1985).

26. Hundhausen, A. J., Burkepile, J. T., and St. Cyr, O. C., J. Geophys. Res. 99, 6543 (1994).

27. Gosling, J. T., Bame, S. J., McComas, D. J., Phillips, J. L., Goldstein, B. E., and Neugebauer, M., Geophys. Res. Lett. 21, 1109 (1994). 